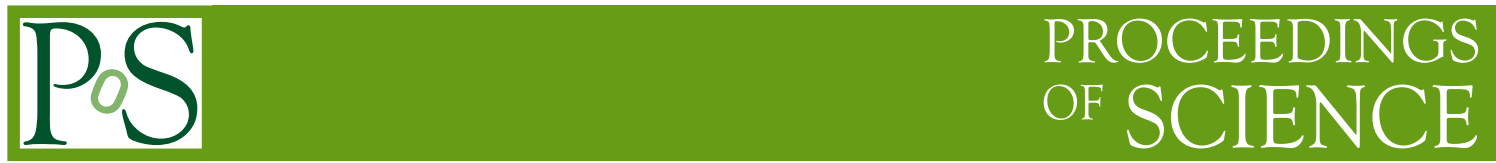

\title{
Multifragment break-up of ${ }^{12} \mathrm{C}$ in photonuclear reactions: a theorist's point of view
}

\section{Igor Pshenichnov*, Andrey Turinge, Vladimir Nedorezov ${ }^{\dagger}$}

Institute for Nuclear Research, Russian Academy of Sciences, 117312 Moscow, Russia

E-mail: pshenich@inr.ru

The absorption of $\sim 1 \mathrm{GeV}$ photons by light and heavy nuclei is simulated by means of the RELDIS model based on the intranuclear cascade and Fermi break-up models. The average excitation energy of residual nuclei created after the photoabsortion on ${ }^{12} \mathrm{C}$ is estimated at the level of 2.5-3 MeV per nucleon, which is essentially higher compared to the photoabsorption on heavy nuclei. It was found that a complete fragmentation of ${ }^{12} \mathrm{C}$ into 12 nucleons is observed typically once per 5000 events. Measured multiplicity distributions of produced fragments are well described by the model. As confirmed by preliminary data obtained by the GRAAL collaboration, multifragment decays of light nuclei can be seen in reactions induced by 700-1500 MeV photons.

XXII International Baldin Seminar on High Energy Physics Problems

15-20 September, 2014

JINR, Dubna, Russia

\footnotetext{
${ }^{*}$ Speaker.

${ }^{\dagger}$ On behalf of the GRAAL collaboration
} 


\section{Introduction}

In proton-nucleus [1] and nucleus-nucleus collisions [2] nuclear matter can be compressed and heated. In this way its properties can be investigated under conditions which are very different from those for nuclei in their ground state, and a phase transition to nucleon gas can be studied [3]. In relativistic or ultrarelativistic heavy-ion collisions [4] tremendous kinetic energy of a projectile nucleus is transfered to collision partners and released in emitted particles. However, even at lower projectile energies of few hundreds $\mathrm{MeV}$ the mass, charge and nuclear structure of the target nucleus also change significantly already at the very beginning of a central or semi-central nucleus-nucleus collision event. This means that hot nuclear systems produced at early stages of such collisions are characterized by large variations of mass and excitation energy. Therefore, such excited nuclei keep only a distant resemblance to the initial projectile or target nucleus because of many nucleons lost or exchanged by collision partners.

It would be interesting to find a way to deliver pure energy instead of energy with some additional nucleons to the target nucleus in order to study decays of a hot nuclear system with definite properties. In the present work the absorption of intermediate-energy ( $\sim 1 \mathrm{GeV})$ photons by light and heavy nuclei is considered for this purpose. It is expected that mass and charge distributions of excited residual nuclei created in photoabsorption are more narrow compared to the corresponding distributions predicted for nucleus-nucleus collisions. Moreover, in contrast to nucleus-nucleus collisions, exited nuclear matter produced in photonuclear reactions is not divided into projectile and target remnants and characterized by much lower angular momentum and collective flow.

The first papers devoted to the disintegration of light nuclei by photons $[5,6,7]$ were published four decades ago. In a recent experiment [8] the disintegration of ${ }^{12} \mathrm{C}$ into four particles by $\sim 40 \mathrm{MeV}$ photons was investigated. However, to the best of our knowledge, no processes of multifragmentation of light nuclei induced by $\sim 1 \mathrm{GeV}$ photons were detected so far. In the present paper we simulate photonuclear reactions with the RELDIS model $[9,10,11]$. The main attention is paid to the disintegration of $700-1500 \mathrm{MeV}$ photons by ${ }^{12} \mathrm{C}$ nuclei and comparison with preliminary data obtained by the GRAAL collaboration for this process.

\section{RELDIS model of photonuclear reactions}

It is widely accepted that inelastic interactions of fast particles with nuclei can be described as a sequence of several stages with a specific model applied at each stage. At the first stage photons of intermediate energy of several hundreds of $\mathrm{MeV}$ interact with an individual nucleons inside the target nucleus. In the present study the RELDIS model [12] is employed to simulate photonuclear reactions by means of the Monte Carlo method.

Simulations of intranuclear cascades involve the production and secondary interactions of mesons as well as recoil nucleons. The quasideuteron absorption of photons and the meson production on intranuclear nucleons are taken into account. The two-body channel $\gamma N \rightarrow \pi N$ dominates up to $E_{\gamma} \sim 0.5 \mathrm{GeV}$, while at $0.5 \leq E_{\gamma} \leq 2 \mathrm{GeV}$ the channels $\gamma N \rightarrow 2 \pi N$ and $\gamma N \rightarrow 3 \pi N$ play the main role. At the end of the hadronic cascade, some quasiparticles remain in the nuclear Fermi gas as holes $\left(N_{h}\right)$, which are knocked-out nucleons, and particles $\left(N_{p}\right)$, which are slow cascade nucleons trapped by the nuclear potential. In the RELDIS model, the excitation energy of the residual 


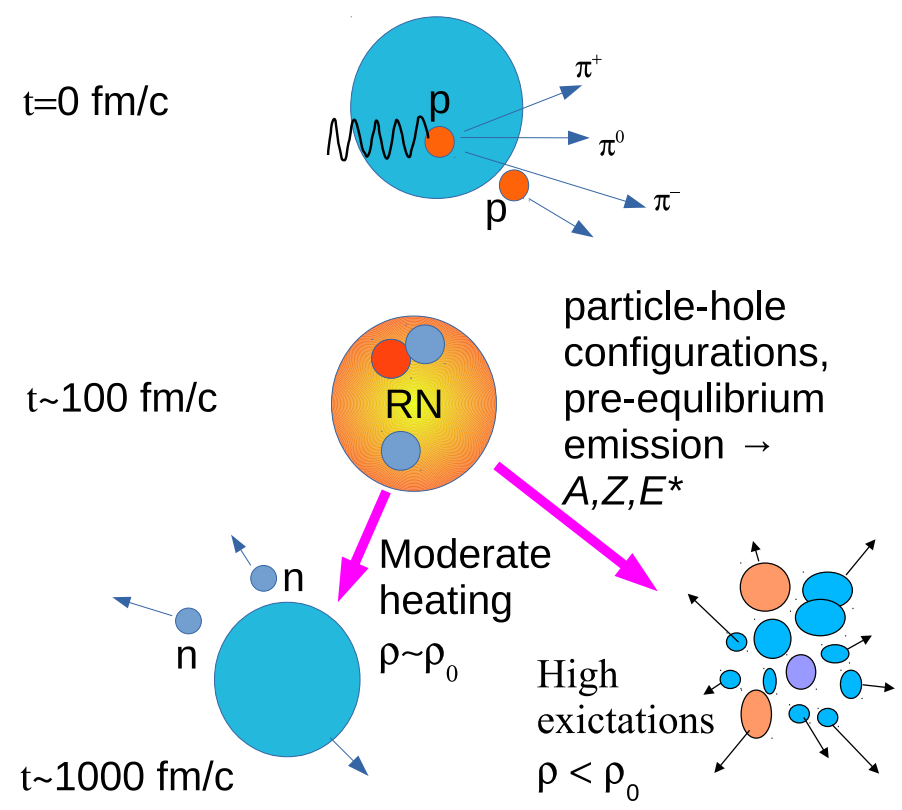

Figure 1: Photonuclear reaction as a multi-stage process represented by a formation of a residual nucleus $(\mathrm{RN})$ as a result of a cascade process up to $\sim 100 \mathrm{fm} / \mathrm{c}$ and a subsequent decay of this nucleus (within $\sim 1000 \mathrm{fm} / \mathrm{c}$ ). Depending on the excitation energy of RN evaporation of nucleons (left) or an explosive decay (right) takes place.

nucleus is defined as

$$
E^{\star}=\sum_{i=1}^{N_{p}} \varepsilon_{i}^{p}+\sum_{i=1}^{N_{h}} \varepsilon_{i}^{h},
$$

where the quasiparticle energies for particles $\varepsilon_{i}^{p}$ and holes $\varepsilon_{i}^{h}$ are measured with respect to the Fermi energy. The numbers of nucleons and protons of the residual nucleus are given by the relations

$$
\begin{aligned}
& A_{R N}=A-\sum_{i=1}^{N_{c}} q_{i}^{c}, \\
& Z_{R N}=Z-\sum_{i=1}^{N_{c}} e_{i}^{c},
\end{aligned}
$$

where $A$ and $Z$ are the numbers of nucleons and protons, respectively, in the target nucleus, and $q_{i}^{c}$ and $e_{i}^{c}$ are the baryon number and charge carried away by the cascade particle $i$.

\section{Photoexcitation of light and heavy nuclei}

As demonstrated [3], an explosive decay of an excited nucleus into three or more nuclear fragments is observed when $E^{\star}$ is comparable to the total binding energy of such a nucleus. This phenomenon of multifragmentation reveals itself at $E^{\star} / A_{R N}$ above $3 \mathrm{MeV}$ per nucleon. RELDIS results for average $E^{\star}$ and $E^{\star} / A_{R N}$ are plotted in Fig. 2 for photons, protons, antiprotons and ${ }^{3} \mathrm{He}$ of different energies interacting with ${ }^{12} \mathrm{C}$ and ${ }^{197} \mathrm{Au}$ nuclei. As seen, the absorption of photons by heavy nuclei leads to relatively low $\left\langle E^{\star} / A_{R N}\right\rangle$. This explains the dominance of evaporation and 
fission of lead and actinide nuclei over their multifragment decays following the absorption of 68 $3770 \mathrm{MeV}$ photons [13]. This was also demonstrated by detecting nuclides with the mass number of 24 - 131 produced in the absorption of bremsstrahlung photons with end-point energies from 300 to $1100 \mathrm{MeV}$ by ${ }^{197} \mathrm{Au}$ [14]. One can also note, see Fig. 2, that photons less effectively heat heavy target nuclei compared to protons, antiprotons and ${ }^{3} \mathrm{He}$.
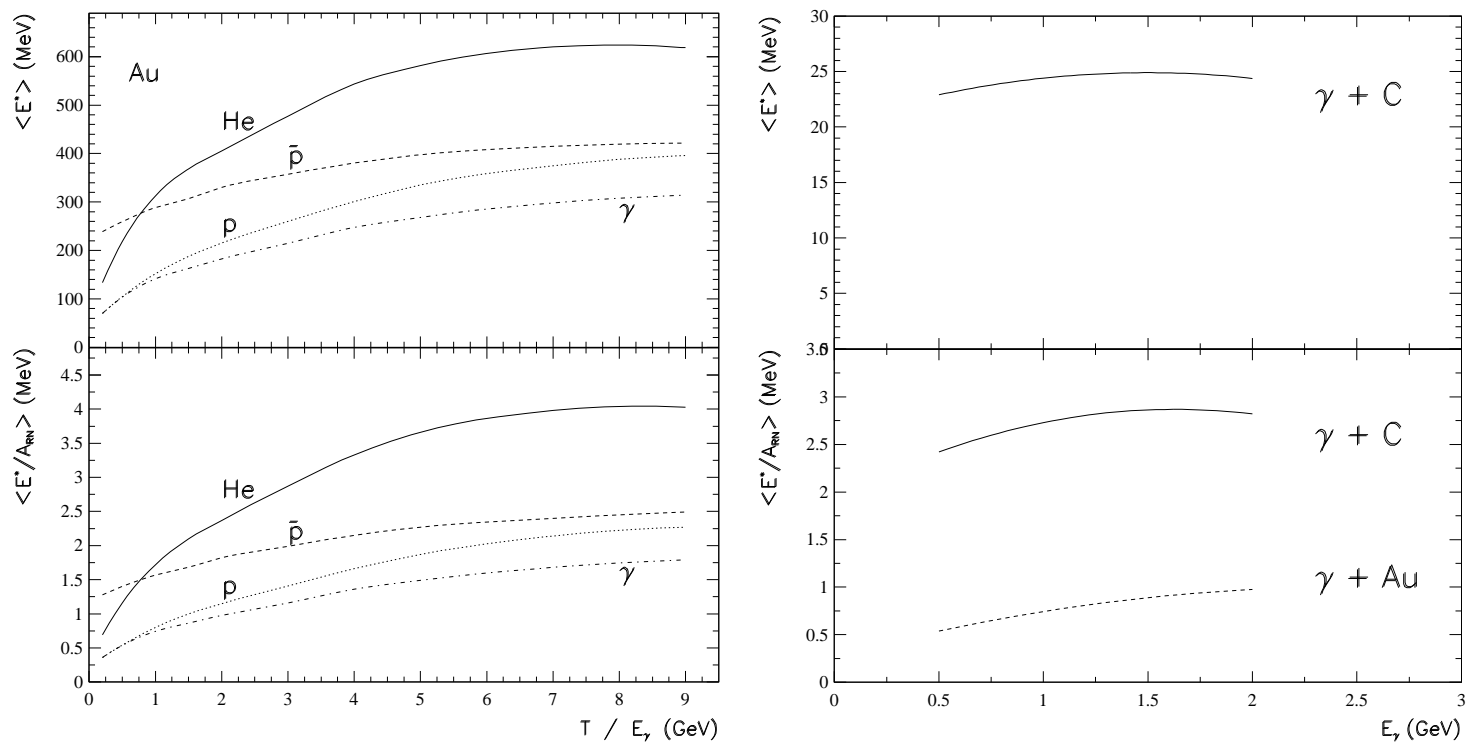

Figure 2: Calculated average excitation energy (top panels) and average excitation energy per nucleon (bottom panels) of residual nuclei resulting from the interaction of photons, protons, antiprotons and ${ }^{3} \mathrm{He}$ with gold nuclei (left) and photons with carbon and gold nuclei (right) as a function of projectile kinetic energy $T$ or photon energy $E_{\gamma}$.

\section{Multi-particle break-up of ${ }^{12} \mathrm{C}$}

Residual nuclei with much higher $\left\langle E^{\star} / A_{R N}\right\rangle$ of $2.5-3 \mathrm{MeV}$ are created in photoabsorption on ${ }^{12} \mathrm{C}$, see Fig. 2. The probability distribution to produce a residual nucleus with a given $E^{\star} / A_{R N}$ is shown in Fig. 3. As seen, the main part of events is characterized by $E^{\star} / A_{R N}<2 \mathrm{MeV}$ with a subsequent emission of individual nucleons by the excited nuclear residue. Nevertheless, there is a low probability to create nuclei with $E^{\star} / A_{R N}>5 \mathrm{MeV}$ which undergo multifragment breakup [3]. In order to describe such decays a model which is similar to the Fermi break-up model for multiple particle production in proton-proton collisions [15] is employed [16, 17, 18]. It is assumed that during the decay time of about $100 \mathrm{fm} / \mathrm{c}$ the excited nucleus disintegrates into cold or slightly excited fragments. The masses of fragments in their ground and lowest excited states are taken from nuclear data tables, and all possible break-up channels, which satisfy the mass number, charge, energy and momenta conservations are considered. For example, an excited ${ }^{12} \mathrm{C}$ nucleus can decay into more than 200 channels containing various combinations of protons, neutrons and light nuclei. It is assumed that the probability of an individual break-up channel containing $n$ particles with masses $m_{i}(i=1, \cdots, n)$ is proportional to its phase space volume $[15,16,17,18]$. The momentum distributions of final products are obtained by the random generation over the 


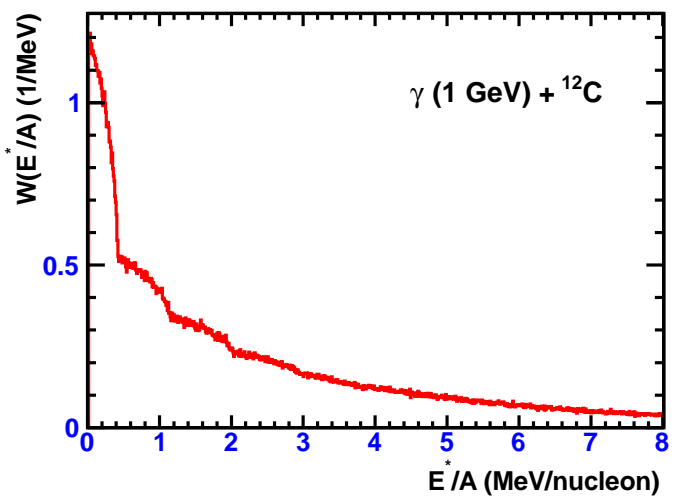

Figure 3: Probability distribution to create a residual nucleus with a given excitation energy per nucleon, $E^{\star} / A_{R N}$, in the absorption of $1 \mathrm{GeV}$ photons by ${ }^{12} \mathrm{C}$, as calculated by the RELDIS model.

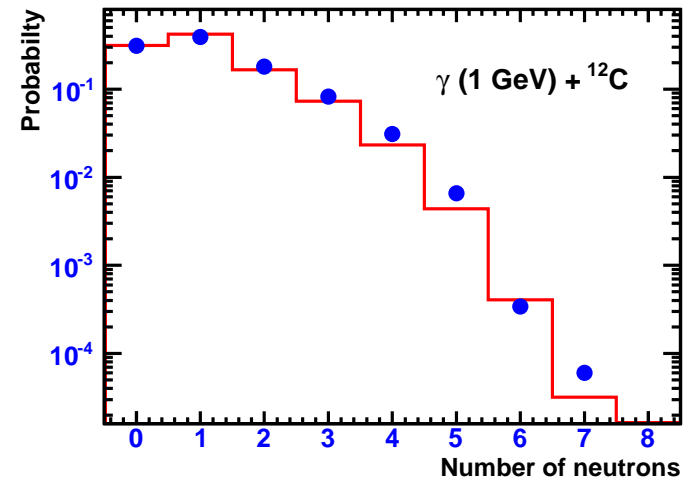

Figure 4: Calculated probabilities of disintegration of ${ }^{12} \mathrm{C}$ by photons with emission of a given number of neutrons (histograms). Preliminary data of the GRAAL collaboration are shown by points.

accessible phase space which is defined by the total energy taking into account the energy and momentum conservation.

The calculated probability distribution of events with a given number of neutrons is shown in Fig. 4. In $\sim 30 \%$ of events protons are emitted without neutrons. Next $\sim 30 \%$ of events are represented by the emission of $1-2$ neutrons and they also correspond to the most probable low excitations. Some $1-2$ neutrons are typically emitted during cascade process. In contrast, highmultiplicity events are very improbable. In particular, events with six neutrons which correspond to a complete disintegration of ${ }^{12} \mathrm{C}$ into individual nucleons are observed once per about 5000 photoabsorption events. It is remarkable that some events with seven neutrons are also observed despite the fact that the target nucleus contains only six neutrons. This is explained by $\gamma p \rightarrow \pi^{+} n$ and other reactions of meson production which lead to a recoil neutron rather than to a proton. A very low probability of these events calculated with the RELDIS model agrees well with the measured value. In general, the calculated distribution is in a good agreement with a distribution measured by the GRAAL collaboration, which is shown in the same figure.

\section{Conclusions}

As follows from our measurements and simulations, the disintegration of ${ }^{12} \mathrm{C}$ nuclei which results from the absorption of 700-1500 MeV photons can be reasonably well described as a twostage process. The RELDIS model predicts a wide distribution of excitation energies of nuclear residues which are created in the photoabsorption after the first cascade stage of the reaction. A large part of the photon momentum is typically taken away by fast cascade particles.

The most probable photodisintegration events are characterized by the emission of 1 or 2 nucleons. However, a complete disintegration of ${ }^{12} \mathrm{C}$ into individual nucleons is also seen in a 
small $(\sim 0.05 \%)$ fraction of photoabsorption events. The model describes the neutron multiplicity distributions very well.

It was found that photons less effectively heat heavy target nuclei compared to protons, antiprotons and ${ }^{3} \mathrm{He}$. However, the average excitation energy of light residual nuclei created after the photoabsortion on ${ }^{12} \mathrm{C}$ is estimated at the level of $2.5-3 \mathrm{MeV}$ per nucleon, which is essentially higher compared to the photoabsorption on heavy nuclei. Some photoabsorption events are associated with $E^{\star} / A_{R N}>5 \mathrm{MeV}$ sufficient for multiframent break-up of ${ }^{12} \mathrm{C}$. This means that multifragment decays of light nuclei can be investigated also in photonuclear reactions in addition to proton-induced reactions and heavy-ion collisions.

\section{Acknowledgments}

I.P. is indebted to Dr. A.S. Botvina for providing the SMM code for simulations of multifragment decays.

\section{References}

[1] A.S. Botvina, A.S Iljinov, I.N. Mishustin, Nucl. Phys. A 507 (1990) 649.

[2] A.S. Botvina, I.N. Mishustin, M. Begemann-Blaich et al., Nucl. Phys. A 584 (1995) 737.

[3] J.P. Bondorf A.S. Botvina, A.S. Iljinov et al., Phys. Reports 257 (1995) 133.

[4] L.P. Csernai, Introduction to relativistic heavy ion collisions, Jonh Wiley \& Sons Ltd., Chichester, England, 1994.

[5] G.G. Taran, Sov. J. Nucl. Phys. 7 (1968) 301.

[6] G.A. Vartapetyan, A.S. Dangulyan, N.A. Demekhina et al., Sov. J. Nucl. Phys. 17 (1973) 356.

[7] V.I. Noga, Yu.N. Ranyuk, P.V. Sorokin, Sov. J. Nucl. Phys. 21 (1975) 243.

[8] S.N. Afanasiev, E.S. Gorbenko, A.F. Khodyachikh et al., Phys. Atom. Nucl. 70 (2007) 839.

[9] I.A. Pshenichnov, I.N. Mishustin, J.P. Bondorf et al., Phys. Rev. C 57 (1998) 1920.

[10] I.A. Pshenichnov, J.P. Bondorf, I.N. Mishustin et al., Phys. Rev. C 64, 024903 (2001).

[11] I.A. Pshenichnov, Phys. Part. Nuclei 42 (2011) 215.

[12] A.S. Iljinov, I.A. Pshenichnov, N. Bianchi et al., Nucl. Phys. A 616 (1997) 575.

[13] I.A. Pshenichnov, B.L. Berman, W.J. Briscoe et al., Eur. Phys. J. A 24 (2005) 69.

[14] H. Haba, M. Igarashi, K. Washiyama et al., J. Nucl. Radiochem. Sci. 1 (2000) 69.

[15] E. Fermi, Prog. Theor. Phys. 5 (1950) 570.

[16] E. Gradsztajn, F. Yiou, R. Klapisch et al., Phys. Rev. Lett. 14 (1965) 436.

[17] A.S. Botvina, Ye.S. Golubeva, A.S. Iljinov, "Statistical simulation of the break-up of light nuclei in hadron-nucleus reactions", Preprint INR P-0657, Moscow, 1990.

[18] A.S. Botvina, A.S. Iljinov, I.N. Mishustin et al., Nucl. Phys. A 475 (1987) 663. 